MATEC Web of Conferences 24,07006 (2015)

DOI: $10.1051 /$ matec conf/ 20152407006

(C) Owned by the authors, published by EDP Sciences, 2015

\title{
Damping and frequency of human-structure interaction system
}

\author{
Ehsan Ahmadi ${ }^{1}$, and Colin Caprani ${ }^{1}$ \\ ${ }^{1}$ Monash University, Department of Civil Engineering, Melbourne, Australia.
}

\begin{abstract}
Human presence can change the dynamic characteristics of human-structure interaction systems, i.e. particularly their damping and frequency. In many design codes, the pedestrian is regarded as a moving force (MF) while a more complete model, referred to as moving spring-mass-damper (MSMD) has received attention recently. Unlike the MF model, the MSMD model is able to take into account the human damping and stiffness effects. This paper is devoted to determining the instantaneous damping and frequency of the human-structure interaction (HSI) system subjected to a single and multiple pedestrians. Each pedestrian is modelled as a MSMD, and a methodology for determining the system damping and frequency properties is described. A simply supported beam structure is considered and modelled in modal space. The imparted pedestrian vertical force is modelled adopting the first four harmonics of Fourier representation using Young's dynamic load factors. It is concluded that human presence can significantly increase and decrease damping and frequency of the structure, respectively and it is so important to consider human effect on the system's damping and frequency in serviceability assessment of footbridges.
\end{abstract}

\section{Introduction}

\subsection{Background}

Human-structure interaction (HSI) plays an important role in the vibration response of slender lightly damped structures. The HSI phenomenon might significantly affect dynamic properties of the structure. Živanović et al. [1] identified modal properties of the HSI system using frequency response functions and showed that the presence of humans on the structure, either in standing or walking form, will increase the damping of the system compared to the empty structure. Živanović et al. [2] revealed that crowd effects can be also modelled as an increase in the damping of the system. Also, Kasperski [3] concluded that a crowd of pedestrians can influence the HSI system's damping using discrete Fourier transform of the acceleration time history response of the bridge.The above-mentioned HSI has been disregarded in design codes such as OHBDC [4], BS 5400 [5], ISO10137 [6], Eurocode 5 [7], Setra [8], and HIVOSS [9]. In other words, these design guides just use a moving force (MF) model that often overestimates the vibration response. However, in biomechanical studies the human body is treated as systems of springs and dampers. Therefore, a human can be modelled as a moving springmass-damper (MSMD) to account for human mass, stiffness, and damping effects on the structural system. The MSMD was introduced by Archbold [10] and [11] into the HSI area. Archbold [11] also found that the MF model is conservative as it does not consider interaction between the pedestrian and the moving bridge, while Clemente et al (2010) [12] showed that if the interaction between the pedestrian and the bridge is to be considered, the pedestrians should be modelled as SMD oscillators.

\subsection{Approach of this study}

In order to model the interaction between a crowd of pedestrians and the bridge, a MSMD model is employed to represent each single pedestrian [13]. This single degree of freedom MSMD model captures changes in the net vertical force applied to the vibrating bridge and is then moved along the bridge at the pedestrian velocity. The bridge used in the model (frequency of $2.0 \mathrm{~Hz}$ and damping ratio of $0.5 \%$ ) is a simply-supported beam, chosen to be susceptible to excitation from typical pedestrian pacing rates. To model the footfall force, a time-varying harmonic force is applied to the bridge at

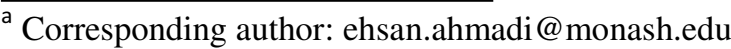


the location of the pedestrian using the Young's dynamic load factors [14] for the first four harmonics. Input parameters for this model include pedestrian mass, step length, pacing frequency, pedestrian stiffness and damping.

To use a MSMD model of the human in a HSI problem, it is required to take reasonable values for the human stiffness and damping ratio. Various values of stiffness and damping have been reported by biomechanical engineers, for example Lee and Farley [15], Arampatzis and Bruggemann [16], Zhang et al. [17], Rapoport et al. [18], Bertos et al. [19], Geyer et al. [20], Lebiedowska [21] for the HSI problem. Caprani et al. [13] used value of 0.3 for human damping ratio (following Zhang et al. [17]), and a wide range of 12-35 $\mathrm{kN} / \mathrm{m}$ for human stiffness. Venuti et al. [22] assumed 0$0.4 \mathrm{kN} / \mathrm{s}$ (as a reasonable range) and $0.2-13 \mathrm{kN} / \mathrm{m}$ (proposed in Bertos et al. [19]) for pedestrian damping coefficient and stiffness, respectively. Zhang et al. [23] proposed human damping ratio of $0.37-0.5$ and frequency of 1.25-1.6 Hz for the subjects considered. Ranges of 1.8$3.5 \mathrm{~Hz}$ and 0.2-0.6 are considered for natural frequency and damping ratio of pedestrians, respectively. There is no distribution for stiffness and damping ratio of the pedestrian in literature and so, a uniform distribution is assumed. Pedestrian mass is represented by a log-normal distribution with a mean of $73.85 \mathrm{~kg}$ and a standard deviation of $15.68 \mathrm{~kg}$ [24]. The step length is taken here to be normally distributed with a mean of $0.66 \mathrm{~m}$ and assuming a coefficient of variation of $10 \%$, a standard deviation of $0.066 \mathrm{~m}$ is used [25]. The phase angle of a pedestrian's vertical harmonic force is taken to be uniformly random distribution in the interval $0-2 \pi$. Pedestrian arrival is considered as a Poisson process and gaps are thus described by the exponential distribution with mean arrival gap of $0.56 \mathrm{~m}$ (Caprani et al. [25]).

The objective of this study is to track frequency and damping of the HSI system during crowd crossing the bridge and see the pair of the bridge frequency and damping at which maximum vibration response is produced. The methodology to track the bridge frequency and damping at each time instant is explained and used.

\section{The HSI system modelling and formulation}

In this study, the HSI system consists of the footbridge structure and a crowd of pedestrians (Figure 1).

The footbridge is modelled as a simply supported beam in modal space. In the MSMD model, the $i$ th pedestrian mass, $m_{p, i}$, is attached to a massless spring and damper with coefficients of $k_{p, i}$ and $C_{p, i}$, respectively as shown in Figure 1. These spring and damper indicate the stiffness and damping of the pedestrian body specifically those acting between the mass centre of the human body and bridge surface. The MSMD model also includes a pulsating force (herein assumed as a harmonic force), which is applied to the bridge surface at the contact point.

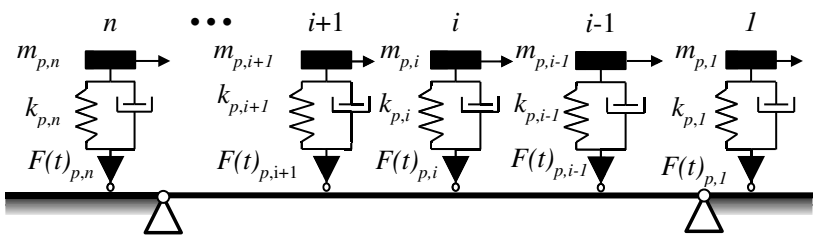

Figure 1. The HSI system used in this study.

To formulate the HSI system, consider a crowd of $n$ pedestrians, with $N$ modes of structural vibration included. The basic equation for the $N+n$ degrees of freedom HSI system in modal-space form is:

$$
\mathbf{M x}+\mathbf{C} \dot{\mathbf{x}}+\mathbf{K x}=\mathbf{F}
$$

in which:

$$
\mathbf{x}=\left\{\begin{array}{l}
\mathbf{q}_{N \times 1} \\
\mathbf{y}_{n \times 1}
\end{array}\right\}
$$

where $\boldsymbol{q}$ is modal coordinate vector of the structure for the $N$ modes; $\mathbf{y}$ is the displacement vector of the $n$ pedestrian from their equilibrium position. Accordingly, the deflection and acceleration responses are:

$$
\begin{gathered}
w(x, t)=\sum_{j=1}^{N} \phi_{j}(x) q_{j}(t) ; \ddot{w}(x, t)=\sum_{j=1}^{N} \phi_{j}(x) \ddot{q}_{j}(t) \\
\mathbf{M}=\left[\begin{array}{cc}
\mathbf{I}_{N \times N} & \mathbf{M}_{12} \\
\mathbf{0}_{n \times N} & \mathbf{m}_{P}
\end{array}\right] \\
\mathbf{C}=\left[\begin{array}{cc}
\mathbf{C}_{11} & \mathbf{0}_{N \times n} \\
\mathbf{C}_{21} & \mathbf{c}_{P}
\end{array}\right] \\
\mathbf{K}=\left[\begin{array}{cc}
\mathbf{K}_{11} & \mathbf{0}_{n \times N} \\
\mathbf{K}_{21} & \mathbf{K}_{P}
\end{array}\right] \\
\mathbf{F}=\left\{\begin{array}{c}
\mathbf{F}_{B} \\
\mathbf{0}_{n \times 1}
\end{array}\right\}
\end{gathered}
$$

The pedestrian-bridge mass coupling matrix, for $i=1, \ldots, n$ , is obtained by:

$$
\mathbf{M}_{12}[1, \ldots, N ; i]=m_{P, i} \boldsymbol{\phi}^{\prime}\left(v_{i} t\right)
$$

The mass-normalized mode shape vector is given by:

$$
\phi^{\prime}(v t)=\left[\frac{\phi_{1}(v t)}{M_{1}}, \ldots, \frac{\phi_{N}(v t)}{M_{N}}\right]^{T}
$$

in which

$$
\phi_{j}(x)=\sin \left(\frac{j \pi v_{i} t}{L}\right) ; M_{j}=\frac{m_{b} L}{2}
$$

The pedestrian mass sub-matrix is:

$$
\boldsymbol{m}_{P}=\operatorname{diag}\left(m_{P, i}\right)
$$


and the pedestrian damping and stiffness matrices, $\boldsymbol{c}_{P}$ and $\boldsymbol{k}_{P}$, are similarly defined. The bridge damping is given by:

$$
\mathbf{C}_{11}=\operatorname{diag}\left(2 \xi_{j} \omega_{j}\right)
$$

and the pedestrian-bridge damping coupling is given as:

$$
\mathbf{C}_{2 l}[i ; 1, \ldots, N]=-c_{P, i} \phi^{T}\left(v_{i} t\right)
$$

The bridge stiffness matrix and pedestrian-bridge stiffness coupling is:

$$
\begin{gathered}
\mathbf{K}_{11}=\operatorname{diag}\left(\omega_{j}^{2}\right) \\
\mathbf{K}_{21}[i ; 1, \ldots, N]=-k_{P, i} \boldsymbol{\phi}^{T}\left(v_{i} t\right)
\end{gathered}
$$

$\phi$ is the non-normalized mode shape vector:

$$
\phi(v t)=\left[\phi_{1}(v t), \ldots, \phi_{N}(v t)\right]^{T}
$$

Finally, the modal force vector for the bridge is given by the total contribution over each pedestrian present, for each mode considered:

$$
F_{B}(t)=\sum_{i=1}^{n} F_{i}(t) \phi^{\prime}\left(v_{i} t\right)
$$

In this study, equation (1) is solved for different number of crowds and first 10 modes of the structure using Nemark-Beta integration.

\section{Eigenvalue analysis of the HSI system}

Herein, an eigenvalue analysis is carried out to obtain frequency and damping matrices of the HSI system in each time. To this end, writing equation (1) in state-space form we obtain:

$$
\dot{\mathbf{v}}=\mathbf{A v}+\mathbf{B}
$$

where:

$$
\mathbf{v}=\left\{\begin{array}{c}
x \\
\dot{x}
\end{array}\right\} ; \mathbf{A}=\left[\begin{array}{cc}
\mathbf{0} & \mathbf{I} \\
-\mathbf{M}^{-1} \mathbf{K} & -\mathbf{M}^{-1} \mathbf{C}
\end{array}\right] ; \mathbf{B}=\left\{\begin{array}{c}
0 \\
\mathbf{M}^{-1} \mathbf{F}
\end{array}\right\}
$$

$\mathbf{A}$ and $\mathbf{B}$ are $2(N+n)$ by $2(N+n)$ and $2(N+n)$ by 1 real matrix and vector, respectively. As the pedestrian walks on the footbridge, the mass, stiffness, and damping matrices of the HSI system change during time. So, the instantaneous modal properties of the HSI system can be obtained using eigenvalue analysis for the entire system. To perform an eigenvalue analysis, the homogenous form of equation (18) (i.e. $\mathbf{B}=\mathbf{0}$ ) must be solved. A convenient approach is to assume a general solution of the following form in vector-space as:

$$
\mathbf{x}=\overline{\mathbf{X}} e^{\lambda t}
$$

in which $\overline{\mathbf{X}}$ is an $N+n$ by 1 complex vector representing the response amplitude and $\lambda$ is the complex quantity. Hence, we have [26]:

$$
\mathbf{v}=\left\{\begin{array}{c}
\overline{\mathbf{X}} \\
\lambda \overline{\mathbf{X}}
\end{array}\right\} e^{\lambda t}=\overline{\mathbf{v}} e^{\lambda t} ; \dot{\mathbf{v}}=\left\{\begin{array}{c}
\lambda \overline{\mathbf{X}} \\
\lambda^{2} \overline{\mathbf{X}}
\end{array}\right\} e^{\lambda t}=\lambda \overline{\mathbf{v}} e^{\lambda t}
$$

Substituting into homogenous form of equation (18) results in:

$$
\lambda \overline{\mathbf{v}}=\mathbf{A} \overline{\mathbf{v}}
$$

This equation represents a generalised eigenproblem whose solution comprises a set of $2(N+n)$ eigenvalues $(\lambda$ vector) that are real or exist in complex conjugate pairs. For the case in which we are interested, i.e., underdamped systems (damping ratios smaller than 1), the values will always appear in complex conjugate pairs. Denoting the eigenvalues by $\lambda$ and $\lambda^{*}$, and the corresponding eigenvectors by $\boldsymbol{\beta}$ and $\boldsymbol{\beta}^{*}$, we have:

$$
\boldsymbol{\psi}=\left\{\begin{array}{c}
\boldsymbol{\beta} \\
\lambda \boldsymbol{\beta}
\end{array}\right\} ; \boldsymbol{\psi}^{*}=\left\{\begin{array}{c}
\boldsymbol{\beta}^{*} \\
\lambda^{*} \boldsymbol{\beta}^{*}
\end{array}\right\}
$$

where $\boldsymbol{\beta}$ and $\boldsymbol{\beta}^{*}$ are the $N+n$ by 1 complex eigenvectors corresponding to the vector-space coordinates $\boldsymbol{x}$. Defining a coordinate transformation for state-space:

$$
\mathbf{v}=\psi^{\prime} \mathbf{Q}(t)
$$

in which $\psi^{\prime}$ is a $2(N+n)$ by $2(N+n)$ complex modal matrix (the state-space modal matrix). $\mathbf{Q}(t)$ stands for modal-space coordinate of the HSI system. Substituting equation (24) into the homogenous form of equation (18) and pre-multiplying by $\psi^{\prime T}$, we obtain:

$$
\psi^{\prime T} \boldsymbol{\psi}^{\prime} \dot{\mathbf{Q}}(t)=\psi^{\prime} \mathbf{A} \boldsymbol{\psi} \mathbf{Q}(t)
$$

Using orthogonality property of eigenvectors, it becomes:

$$
\dot{\mathbf{Q}}(t)=\mathbf{a Q}(t)
$$

in which $\boldsymbol{a}$ is a diagonal $2(N+n)$ by $2(N+n)$ matrix. Thus, equation (26) shows a set of $2(N+n)$ uncoupled equations which is equivalent to having a set of independent $2(N+n)$ SODF systems. In other words, there are a set of $N+n$ complex eigenvalues $\lambda$ and the corresponding $N+n$ complex eigenvectors $\phi$ plus another set of $N+n$ complex eigenvalues $\lambda^{*}$ and the corresponding $N+n$ complex eigenvectors $\phi^{*}$. Using free vibration theory of SDOF systems, eigenvalue for mode $j$ of the HSI system is written under the form:

$$
\lambda_{j}=-\omega_{j} \xi_{j}+i \omega_{j} \sqrt{1-\xi_{j}^{2}}
$$


where $\omega_{j}$ and $\xi_{j}$ stand for circular frequency and damping ratio of mode $j$. It should be noted that equation (27) is for underdamped systems, i.e. $\xi_{j}<1$. The absolute value of eigenvalue is obtained from:

$$
\left|\lambda_{j}\right|=\left(-\omega_{j} \xi_{j}\right)^{2}+\left(\omega_{j} \sqrt{1-\xi_{j}^{2}}\right)^{2}=\omega_{j}
$$

As a result, the frequency and damping ratio of mode $j$ are determined from:

$$
f_{j}=\frac{\left|\lambda_{j}\right|}{2 \pi} ; \xi_{j}=\frac{\left|\operatorname{Re}\left(\lambda_{j}\right)\right|}{\left|\lambda_{j}\right|}
$$

To sum up, first eigenproblem for the HSI in the statespace is solved. The frequency and damping ratio of all the $N+n$ modes can be obtained using equations (28) and (29). Since we are dealing with the HSI system under a crowd of pedestrians, the main issue is to identify the bridge frequency and damping among a large number of values. The next section represents an algorithm to find bridge frequency and damping.

\section{Tracking algorithm for a crowd of pedestrians}

The HSI system has many DOFs under a crowd of pedestrians. Therefore, it is not easy to isolate the bridge frequency and damping ratio from the large number of frequency-damping pairs (see Figure 2 for example). For this purpose, the minimum Euclidean norm of the bridge frequency and damping ratio vector between two consecutive times, $t$ and $t+\Delta t$ is used to track the evolution of the parameters. Denoting $f(t)$ and $\zeta(t)$ as a frequency and damping pair at time $t$. The algorithm commences with the known properties of the empty bridge for $t_{0}=0$. The subsequent tracking in twodimensional space is then defined by:

$$
\mathbf{h}_{p}\left(t_{i}\right)=\min _{j}\left\|\mathbf{h}_{j}\left(t_{i}\right)-\mathbf{h}_{k}\left(t_{i-1}\right)\right\|
$$

in which:

$$
\mathbf{h}_{p}\left(t_{i}\right)=\left[f\left(t_{i}\right), \zeta\left(t_{i}\right)\right]^{T}
$$

is the vector of bridge frequency and damping found at the current time step to be at system DOF $p$; and

$$
\mathbf{h}_{k}\left(t_{i-1}\right)=\left[f\left(t_{i-1}\right), \zeta\left(t_{i-1}\right)\right]^{T}
$$

is the vector of bridge frequency and damping known from the previous time step to be at system DOF $k$, and

$$
\mathbf{h}_{j}\left(t_{i}\right)=\left[f_{j}\left(t_{i}\right), \zeta_{j}\left(t_{i}\right)\right]^{T}
$$

is the vector of frequency-damping pair $j \in\{1, \ldots, N+n\}$ from the $N+n$ HSI system DOFs, found from equation (30) at the current time step, $t_{i}$.

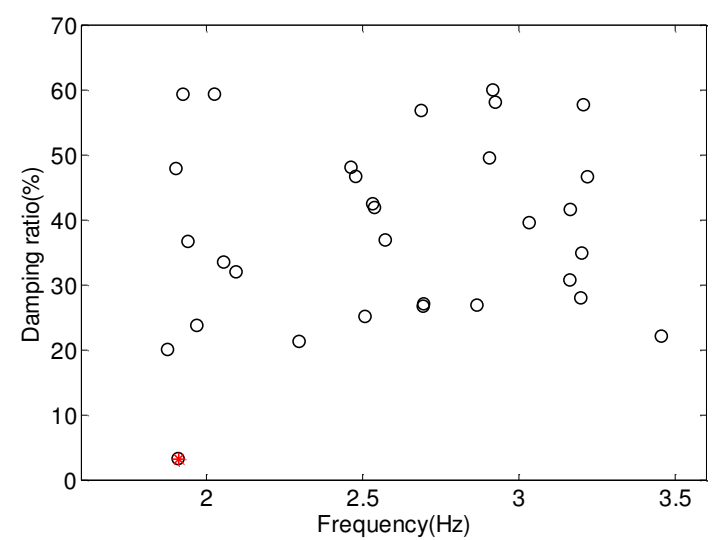

Figure 2. Tracking sample for a random crowd of 30 pedestrians $(n=30)$.

In Figure 2, a tracking sample is shown for a crowd of 30 pedestrians $(n=30)$. In the modal analysis herein, the first 10 modes of the structure are considered in the modal analysis. Thus, based on the previous section, the eigenvalue analysis of the HSI system results in 40 different frequency and damping pairs (in fact, 80 pairs which each two pairs are the same due to complex conjugate pairs of eigenvalues). Higher modes of the empty bridge correspond to $8,18, \ldots\left(k^{2} f_{b}, k=2,3, \ldots\right)$ which is too far from the fundamental frequency and has not been shown in Figure 2. 31 pairs left are shown by a dark circle at the time of $15 \mathrm{~s}$ while the frequency and damping ratio for the bridge corresponding to the previous time instant (14.99 s) is illustrated by a red star. As can be seen, due to the slight change in frequency and damping between two consecutive time instants, the algorithm can well track damping and frequency of the bridge through time. Figure 3 shows the entire bridge frequency and damping pairs for the 30 pedestrians random crowd crossing the bridge (Figure 2). The shape is like a closed loop and the pedestrians enter the bridge, increase the damping and decrease the frequency. Afterwards, when they leave the bridge, the frequency and damping approach those of empty bridge $\left(\mathrm{f}_{\mathrm{b}}=2 \mathrm{~Hz}\right.$, $\zeta=0.5 \%$ ). The arrows show the direction of closed loop which in this case is counter clockwise. Also, the red point indicates the frequency and damping pair considered in Figure 2. 


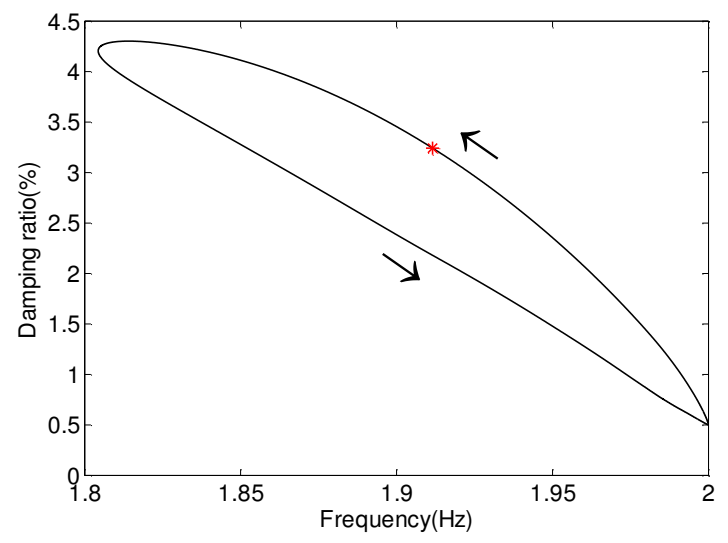

Figure 3. Bridge frequency and damping ratio pairs for a random crowd of 30 pedestrians $(n=30)$.

\section{The HSI effects on the bridge frequency and damping}

In order to consider crowd effects on vibration response, it is better to first see how a deterministic crowd of pedestrians can change bridge frequency and damping and accordingly vibration response. To this end, weight, step length, frequency, and damping of each pedestrian are taken as $75 \mathrm{~kg}, 0.6 \mathrm{~m}, 1.8 \mathrm{~Hz}$, and 0.4 , respectively. The gap between two adjacent pedestrians is assumed constant at $1.0 \mathrm{~m}$. Figure 4 illustrates the results for a pacing frequency of $2.0 \mathrm{~Hz}$, the same as the empty bridge frequency, and a crowd of 30 pedestrians. The bridge reaches its minimum frequency and maximum damping at half the total event time when the maximum number of pedestrians are on the bridge. Interestingly, the maximum vibration response occurs at the beginning and end of the time history which corresponds to high frequency and low damping for the bridge. At first, since the bridge frequency is very close to pacing frequency, the resonance phenomenon starts building up. However, the vibration response drops as the bridge frequency deviates from the pacing frequency due to the human-structure interaction. Again, at the end, the pedestrians move off the bridge and bridge frequency start approaching the pacing frequency and consequently the build-up resonance phenomenon occurs. This means that a crowd of pedestrian can change the build-up resonance phenomenon as it changes bridge frequency.

To explain more, the vibration response is shown in Figure 5 for pacing frequency of $1.85 \mathrm{~Hz}$ which corresponds to the minimum bridge frequency. As can been seen, the vibration response trend is different from Figure $4 \mathrm{a}$ and the peak vibration response happens at the half the crossing time which coincides with the minimum frequency and maximum damping for the bridge. To sum up, it is concluded that the bridge frequency due to the HSI dominates the system vibration response and the pair of bridge frequency and damping at which maximum response happens, can change accordingly.

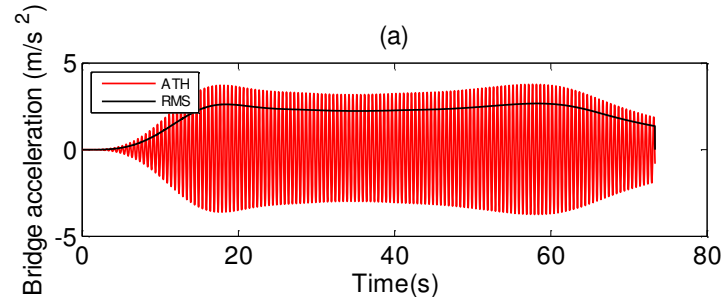

(b)

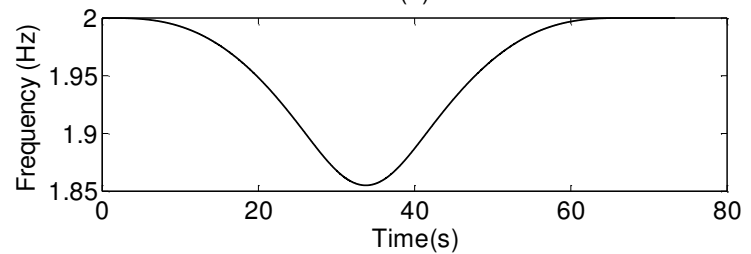

(c)

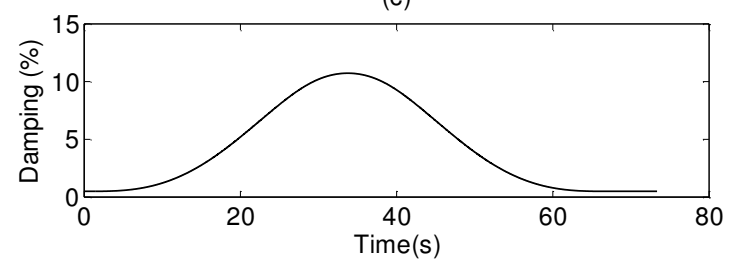

Figure 4. Bridge: (a) acceleration time history (ATH) and root mean square (RMS) response, (b) frequency, and (c) damping for pacing frequency of $2.0 \mathrm{~Hz}$ and a crowd of 30 pedestrians.

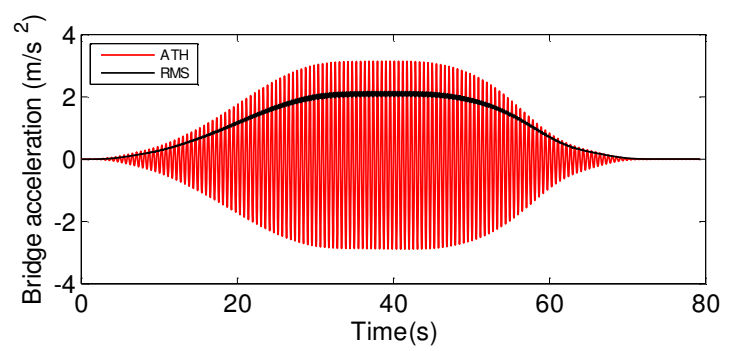

Figure 5. Bridge acceleration response for pacing frequency of $1.85 \mathrm{~Hz}$ and a crowd of 30 pedestrians

\section{Frequency and damping corresponding to maximum response}

To deepen the preceding analysis, the maximum RMS response, and corresponding bridge frequency and damping subjected to a single pedestrian with random properties for 1000 simulations are shown in Figure 6 . First of all, the number of vibration responses larger than $1 \mathrm{~m} / \mathrm{s}^{2}$ is very low and majority of responses are smaller than $1 \mathrm{~m} / \mathrm{s}^{2}$. The bridge damping when subjected to a single pedestrian significantly changes, whereas its frequency change is negligible due to small human-bridge mass ratio. The pair of bridge frequency and damping extremely alters from one simulation to another. 


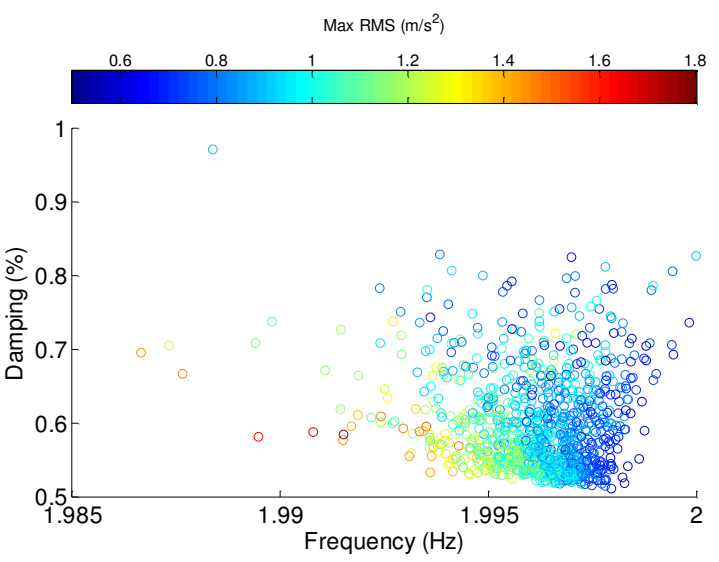

Figure 6. Maximum RMS response and corresponding bridge frequency and damping for a single pedestrian with random parameters.

Figures 7 and 8 illustrate the maximum RMS response, and corresponding bridge frequency and damping subjected to a crowd of 10 , and 30 pedestrians with 1000 simulations. The range of RMS response is close to single pedestrian. However, intuitively, we expect that the vibration response due to a crowd must be much larger than a single pedestrian. It means that crowds effect (especially its damping) can be beneficial and help moderate vibration response. As can be seen, the maximum response happens at different pairs of bridge frequency and damping for random crowds. As the crowd becomes denser, the bridge frequency and damping values corresponding to maximum response have less dispersion. Interestingly, the bridge frequency and damping change approximately follow a roughly linear trend and this can clearly be observed in Figure 8.

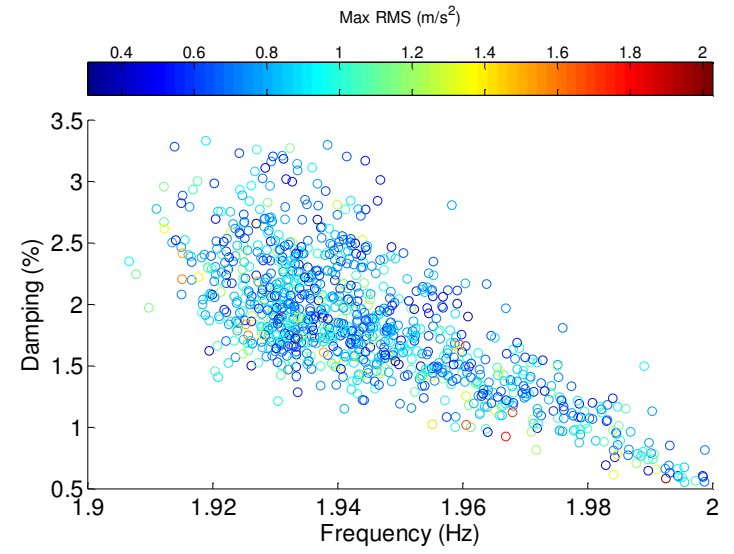

Figure 7. Maximum RMS response and corresponding bridge frequency and damping for a crowd of 10 pedestrians.

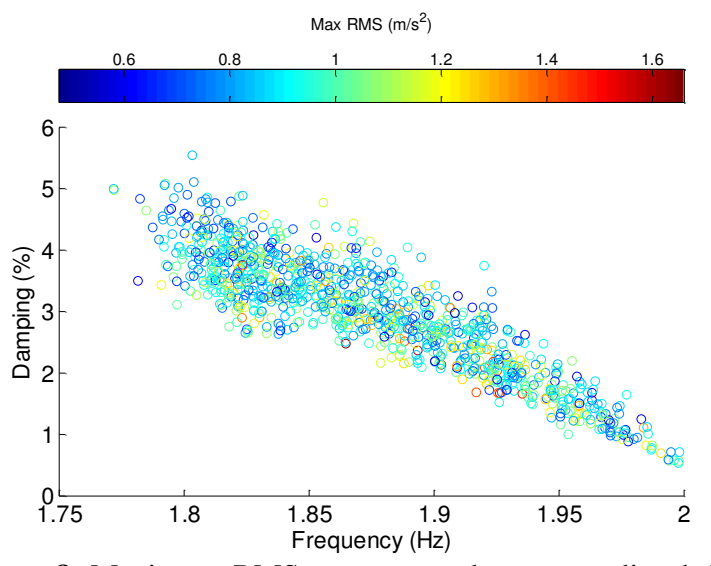

Figure 8. Maximum RMS response and corresponding bridge frequency and damping for a crowd of 30 pedestrians.

As mentioned previously, depending on the pacing frequency and its proximity to the instantaneous bridge frequency, the maximum response can be achieved. Since, pacing frequency is chosen randomly based on a normal distribution for each pedestrian, the frequency at which maximum response occurs depends on the number of pedestrians who have a pacing frequency very close to the bridge frequency at a specific time instant. On the other hand, pedestrians damping and stiffness distribution in the crowd can affect the capability of a crowd in moderating the vibration response. This effect can in turn change the location of maximum response as well.

\section{Conclusion}

In this study, the frequqnecy and damping of the HSI system subjected to crowds of pedestrians is investigated. Each pedestrian is modelled as a spring-mass-damper. The bridge is modelled as a simply supported beam in modal space. The eigenvalue analysis is carried out to obtain the frequency and damping matrices of the HSI system in each time. In order to isolate bridge frequency and damping from all DOFs, a tracking methodolgy is used.

It is concluded that the bridge frequency and damping significantly change due to the interaction between the bridge and a crowd of pedestrians can change the buildup resonance phenomenon as it changes bridge frequency. Also, the pair of bridge frequency and damping at which maximum vibration response occurs is not necessarily the minimum frequency and maximum damping.

\section{References}

[1] S. Živanović, I.M. Diaz, A. Pavić, Influence of walking and standing crowds on structural dynamic properties, in: 27th IMAC Conf., Orlando, USA, 2009.

[2] S. Živanović, A. Pavić, E.T. Ingólfsson, Modeling Spatially Unrestricted Pedestrian Traffic on Footbridges, J. Struct. Eng. 136 (2010) 1296-1308. doi:10.1061/(ASCE)ST.1943- 
[3] M. Kasperski, Damping induced by pedestrians, in: 9th Int. Conf. Struct. Dyn. EURODYN,Porto, Port. 30 June - 2 July, 2014: pp. 1059-1064.

[4] OHBDC, Ontario Highway Bridge Design Code, Highway Engineering Division, Ministry of Transportation and Communication, Ontario, Canada, (1983).

[5] Steel, Concrete and Composite Bridges-Part 2: Specification for Loads; Appendix C: Vibration Serviceability Requirements for Foot and Cycle Track Bridges, BS 5400. UK: British Standards Association, London, (1978).

[6] ISO 10137 - Bases for design of structures Serviceability of buildings and walkways against vibrations, (1992).

[7] Eurocode 5, Design of Timber Structures-Part 2: Bridges, ENV 1995-2: 1997, European Committee for Standardization, Brussels, Belgium, (1997).

[8] Sétra, Guide methodologique passerelles pietonnes (Technical guide Footbridges: assessment of vibrational behaviour of footbridges under pedestrian loading), 2006.

[9] Design of footbridges - HIVOSS (Human Induced Vibrations of Steel Structures), 2008.

[10] P. Archbold, Interactive Load Models for Pedestrian Footbridges, PhD Thesis, University College Dublin, 2004.

[11] P. Archbold, Evaluation of novel interactive load models of crowd loading on footbridges, in: 4th Symp. Bridg. Infrastruct. Res. Ireland, Natl. Univ. Ireland, Galw., 2008: pp. 35-44.

[12] P. Clemente, G. Ricciardi, F. Saitta, DYNAMIC RESPONSE OF A FOOTBRIDGE TO WALKING PEOPLE, in: Int. Work. Civ. Struct. Sealth Monit. (Weigh Motion), Load Capacit. Bridg. Performance, Italy, 2008.

[13] C.C. Caprani, J. Keogh, P. Archbold, P. Fanning, Characteristic vertical response of a footbridge due to crowd loading, in: Eurodyn 2011 - Eigth Eur. Conf. Struct. Dyn., Leuven, Belgium, 2011: pp. 978-985.

[14] P. Young, Improved floor vibration prediction methodologies. ARUP vibration seminar, (2001).

[15] C.R. Lee, C.T. Farley, Determinants of the center of mass trajectory in human walking and running., J. Exp. Biol. 201 (1998) 2935-2944.

[16] A. Arampatzis, G.P. Brüggemann, V. Metzler, The effect of speed on leg stiffness and joint kinetics in human running., J. Biomech. 32 (1999) 1349-53.

[17] L. Zhang, D. Xu, M. Makhsous, F. Lin, Stiffness and viscous damping of the human leg, in: 24th Annu. Meet. Am. Soc. Biomech., Chicago, 2000.

[18] S. Rapoport, J. Mizrahi, E. Kimmel, O. Verbitsky, E. Isakov, Constant and variable stiffness and damping of the leg joints in human hopping., 2003. doi:10.1115/1.1590358.

[19] G.A. Bertos, S.M. Ieee, D.S. Childress, M. Ieee, S. a Gard, The vertical mechanical impedance of the locomotor system during human walking with applications in rehabilitation, in: Proc. 9th Int. Conf. Rehabil. Robot., 2005: pp. 380-383.

[20] H. Geyer, A. Seyfarth, R. Blickhan, Compliant leg behaviour explains basic dynamics of walking and running., Proc. Biol. Sci. 273 (2006) 2861-7. doi:10.1098/rspb.2006.3637.

[21] M.K. Lebiedowska, T.M. Wente, M. Dufour, The influence of foot position on body dynamics., J. Biomech. $\quad 42 \quad$ (2009) 762-6. doi:10.1016/j.jbiomech.2008.12.021.

[22] F. Venuti, V. Racic, A. Corbetta, Pedestrianstructure interaction in the vertical direction:Coupled Oscillator-Force Model for Vibration Serviceability Assessment, in: Proc. 9th Int. Conf. Struct. Dyn. EURODYN 2014, Porto, Portugal, 2014: pp. 915-920.

[23] M. Zhang, C.T. Georgakis, W. Qu, J. Chen, C. Engineering, SMD model parameters of pedestrians for vertical human-structure interaction, in: IMAC XXXIII A Conf. Expo. Struct. Dyn., 2015.

[24] K. Portier, J.K. Tolson, S.M. Roberts, Body weight distributions for risk assessment., Risk Anal. 27 (2007) 11-26. doi:10.1111/j.15396924.2006.00856.x.

[25] C.C. Caprani, J. Keogh, P. Archbold, P. Fanning, Enhancement factors for the vertical response of footbridges subjected to stochastic crowd loading, Comput. Struct. 102-103 (2012) 87-96. doi:10.1016/j.compstruc.2012.03.006.

[26] M. Silva, H. Lieven, L. Shingle, T. Urgueira, Theoretical and Experimental Modal Analysis, Res. Stud. Press LTD, Baldock, Hertfordshire, Engl. (1998). 